\title{
Introduction: Special Issue on Evolutionary Dynamics and Agent-Based Modeling in Economics
}

\author{
Herbert Dawid ${ }^{1} \cdot$ Andreas Pyka ${ }^{2}$ \\ Published online: 4 July 2018 \\ (C) Springer Science+Business Media, LLC, part of Springer Nature 2018
}

This special issue on "Evolutionary Dynamics and Agent-based Modeling in Economics" includes a set of papers which demonstrate the recent progress in the methodology and application of computational agent-based models in economics and also highlight the potential of agent-based approaches for addressing main topics in evolutionary economics, like the (innovation driven) dynamics of industries and markets or the evolution of norms. The special issue also features agent-based analyses of other highly topical issues, in particular the role of debt and financial constraints for economic dynamics and the interplay between economic growth, environmental policy and climate change. A large part of the papers included in this special issue have been presented at the annual meetings in 2014 and 2015 of the Committee for Evolutionary Economics of the Verein für Socialpolitik, the association of economists in Austria, Germany and Switzerland. The committee has been active in fostering research and exchange of ideas in the area of Evolutionary Economics for more than 25 years. Over these years agent-based approaches have gained more and more importance for the research of the members of the committee. This special issue is an acknowledgment of this development and at the same time intends to inspire new contributions in this important area of research.

One of the main challenges agent-based modelers face is bringing their models to the data through estimation or calibration. The computational effort induced by simulating the model extensively at each point during a systematic search in a (often large) parameter space as well as conceptual questions related measuring the goodness of fit of the model output with the data and issues of rugged and flat landscapes make this a hard problem. The first paper in this special issue by Chen and Lux contribute to the growing literature on this issue by exploring the performance of

$\bowtie$ Herbert Dawid

hdawid@wiwi.uni-bielefeld.de

Andreas Pyka

a.pyka@uni-hohenheim.de

1 Department of Business Administration and Economics and Center for Mathematical Economics, Bielefeld University, Bielefeld, Germany

2 Institute of Economics, Chair of Innovation Economics, Hohenheim University, Stuttgart, Germany 
a simulated methods of moments (SMM) approach for the estimation of a financial market model incorporating fundamentalist and noise trader who switch between being optimistic and pessimistic. A main insight from their study is that due to the shape of the objective function a global grid search followed by systematic SMM is needed to deliver satisfactory results of the estimation.

One of the major opportunities of ABM is to go beyond the equilibrium view prevailing in many fields in mainstream economics. In particular, when different development stages are typical, economists fall back to the idea of stylized facts to highlight empirical regularities for single stages. These stylized facts are then addressed individually to be explained in theoretical models. ABMs instead allow for a dynamic view and with it a comprehensive treatment of stylized facts. The second paper in this special issue by Brenner and Duschl focuses on the rich empirical dynamics observed for industry evolution and their particularities concerning firm growth, industry classification as well as stage of the industry life cycle. A wide applicability of this model is given as the authors work out specific parameter ranges for the reproduction of single stylized facts instead as suggesting different models for their explanation.

Also the contribution of Vermeulen and Pyka presents an agent-based model which is carefully linked to empirical stylized facts. They develop a sophisticated model of technological discoveries, which takes into account the knowledge structure on which discoveries are based. They represent this structure as a knowledge graph and empirically calibrate it using data from the European Patent Office. The calibrated model is used to analyze in a multi-regional setting how the interplay between the distribution of local knowledge across different fields and the topology of spatial networks, connecting agents in different regions, determines the innovativeness of the regions. They highlight the positive role of gatekeepers, which ensure connections between different regions, for the speed of technological progress.

Whereas Vermeulen and Pyka consider the determinants of innovativeness of an economy, the paper by Heinrich explores in an agent-based model the diffusion of (product or technological) standards in an economy. In particular, an industry with two segments is considered where in each segment two standards compete. Standards are tied, in a way such that (positive) network externalities across segments for tied standards arise. Heinrich considers a replicator dynamic for describing the evolution of the shares of the standards as well as an agent-based model, assuming that individual adoption decisions are based on observations in the neighborhood of an agent and these neighborhoods are expressed through different network structures. Apart from studying the effect of these different network structures on the dynamics of standard shares the agent-based model also allows to examine the importance of putting initial adopters in different positions of the network.

The paper by $\mathrm{A} ß$ muth introduces an $\mathrm{ABM}$ which addresses the problem of financing innovation. Obviously, financial markets are confronted with the major problem of Knightian Uncertainty when it comes to the decision to invest in innovative ventures exploring new knowledge fields and their potential economic applications. Due to the most important role of uncertainty for this decision, only numerical models can be meaningfully applied, as the phenomenon is out of scope for optimization approaches. Additionally, we want to mention, that Aßmuth explicitly refers to Nelson and Winters (1982) Evolutionary Theory of Economic Change concerning the design of his model. 
Nelson and Winter began with simulations in evolutionary economics and this special issue is a more than 30 years later consequence of their starting shot.

One of the most devastating experiences in the first decade of the twenty-first century was the financial crisis, which was not forecasted by economists. Although economics was considered the approach to explain crises at the turn of the twentieth century, crises are no longer an endogenous possibility to mainstream economic theories one century later. A fatal error, as it turned out. In the paper of van der Hoog crises return as an endogenous phenomenon in an ABM. The paper underlines the creative potential of this class of models, highly suited to generate most required insights to improve our understanding of complex phenomena challenging economic development.

Geisendorfs paper also focuses on one of the grand anthropogenic challenges of the early twenty-first century. This time not out of control financial markets with devastating feedbacks on real economic development are addressed, but climate change and the threatening non-sustainability of our contemporary economic system. Like for the other papers in this special issue, the specific advantage of ABM here is the explicit focus on complex phenomena, which are to be expected in all kind of intertemporal relationships. Specifically, Geisendorf develops an ABM which reproduces the results of an early forerunner model in this literature. As her conclusion, Geisendorf recommends the further application of this class of models in terms of a model to model way of thinking.

The flexibility of ABMs concerning their application in interdisciplinary contexts is unquestioned. In particular, the design of the model's environment allows the consideration of e.g. industrial specificities (see also the contribution of Brenner and Duschl in this special issue) which goes beyond other methodological alternatives. Beckenbach, Daskalakis and Hofmann make use of this flexibility, and, like Geisendorf, focus on complex phenomena from human-nature-interaction. They address energy production and the increasing replacement of fossil-based and nuclear technologies by renewables. With their framework they generate an $\mathrm{ABM}$ tool for experiments to discover conditions for, and impacts of different development paths, which in turn depend on and can be influenced by regulation.

Also the paper by Roos considers how the dynamics of environmental damage and climate change is linked to economic policy and economic dynamics. In particular, Roos develops an endogenous growth model encompassing carbon emissions and environmental variables as well as endogenously evolving societal values. The societal values, whose dynamics are driven by the evolution of GDP and of the state of the environment, determines the allocation of investment in the economy to more or less environmentally friendly activities. Using a baseline calibration the model produces outcomes corresponding to quite plausible ranges of temperature increase and economic growth.

In the last paper of this special issue by Duca, Helbing and Nax, the authors emphasize a further advantage of ABMs for developing new insights in economics and more general in social sciences by getting rid of the tight straitjacket of conventional methodologies. The authors address the theoretically most interesting case of voluntary contribution games, which addresses a most relevant social dilemma we are encountering in many critical situations. The opportunities to address this phenomenon with agent-based models are richer than with the prevailing methodology of standard game 
theory. Heterogeneity of agents and the variability of interactions are the ingredients of adaptive complex systems, which require for their analysis the modelling flexibility of the agent based modelling approach.

We wish to thank Hans M. Amman, Editor-in-Chief of Computational Economics, for giving us the opportunity to edit this special issue and the staff of the journal for their technical support. We also would like to thank the authors who have contributed to this volume submitting their work and all the reviewers as well as the participants of the two annual meetings of the Committee for Evolutionary Economics for their input on the submitted papers. 